\title{
SPATIAL MAPPING OF THE THERMOSPHERIC NEUTRAL WIND FIELD
}

\author{
C. A. TEPLEY*, R. G. BURNSIDE, J. W. MERIWETHER, Jr. and P. B. HAYS \\ Space Physics Research Laboratory, Department of Atmospheric and Oceanic Science, The University of \\ Michigan, Ann Arbor, MI 48109, U.S.A. \\ and \\ L. L. COGGER \\ Department of Physics, The University of Calgary, Calgary, Alberta T2N 1N4, Canada
}

(Received in final form 22 August 1983)

\begin{abstract}
$\Lambda$ bstract There are many possible observing strategies available for mapping the thermospheric wind ficld by using observations of the Doppler shift of the $O\left({ }^{1} \mathrm{D}\right)$ airglow with a Fabry-Perot interferometer. The determination of the neutral wind field from observed line-of-sight velocities invariably involves some assumptions about the nature of the wind field. A standard method of observing employs the assumption that horizontal gradients in the wind field are linear. An analysis of measurements from Arecibo, Puerto Rico, that makes use of this assumption, is discussed. For work at high latitudes this assumption may be unrealistic. An alternative approach that requires that local time and longitude be interchangeable, but eschews the assumption of linear gradients has been developed and used at Ann Arbor, Michigan, and Calgary, Alberta. We examine these different techniques, and illustrate the discussion with some typical results.
\end{abstract}

\section{INTRODUCTION}

Measurements of thermospheric neutral winds have now been made for over a decade by using Fabry-Perot interferometers to monitor the Doppler shifts of the atomic oxygen $O\left({ }^{1} \mathrm{D}\right)$ airglow emission at $6300 \AA$ [Hernandez and Roble, 1979; Hernandez, 1980; Meriwether, 19837. Until the recent launch of the Dynamics Explorer satellites, this work was groundbased. The basic challenge for ground-based Fabry-Perot interferometry is to obtain as much information as possible about the thermospheric neutral wind field using the line-of-sight velocities observed from emitting regions located anywhere within a horizontal range of $750 \mathrm{~km}$ from the instrument.

To obtain a complete description of the wind velocity, $\mathbf{U}$, it would be necessary to measure all three components of $\mathbf{U}$ simultaneously at a specified location. Clearly this is not possible using a single station. However, our goal may be approached if some physically reasonable assumptions about the variation of $\mathbf{U}$ with latitude and longitude are introduced. In most analysis methods it is also necessary to assume that the vertical component of $\mathbf{U}$ is small compared to

*Now at Arecibo Observatory, P.O. Box 995, Arecibo, PR 00613, U.S.A. the horizontal component. We shall refer to this as the 'standard approach'.

Many authors have made use of the traditional method of observing in the four cardinal directions (i.e. $\mathrm{N}-\mathrm{E}-\mathrm{S}-\mathrm{W}$ ) using a scanning mirror system (see, for example, Hays and Roble [1971a], Hernandez and Roble [1976] and Sipler and Biondi [1979]). The mean overhead meridional velocity component is then found by taking the difference between the North and South measurements. The zonal component is found in a similar manner. This method implicitly incorporatcs what we call the standard approach. This is because it is necessary to assume that the spatial variation of $\mathbf{U}$ is linear over the region observed. If the instrument is stable and well calibrated against a spectral lamp or a laser, measurements of the vertical component of $\mathbf{U}$ are also possible [Hernandez, 1982].

Scientists working in the radar field have made use of a 'heam-swinging' technique to measure line-of-sight velocities as a function of azimuth [Browning and Wexler, 1968; Hagfors and Behnke, 1974]. A harmonic series is fit to the measurements lo determine the wind field. This method has also been applied by Burnside $e t$ al. [1981] to Fabry-Perot observations of the $6300 \AA$ nightglow where the instrument viewed the sky at $60^{\circ}$ zenith angle in eight azimuth positions. This method is not conceptually different from the standard approach. However, the availability of eight independent measurements allows one to give less weight to any 
poor measurements when performing the harmonic analysis.

For measurements at magnetic latitudes greater than about $60^{\circ}$, some argument may exist about what assumptions really are physically reasonable. Within the auroral zone, the thermospheric winds are known to have much more pronounced spatial variations than are found at lower latitudes (Killeen et al., 1982). For this reason, we have investigated a diflerent method of analysis that makes no assumptions about the spatial gradients in $\mathbf{U}$, but instead assumes that local time and longitude are interchangeable. Such a method was used by Smith et al. [1982] to determine ion velocities from observations of the $\mathrm{O}^{+}$emission at $7320 \AA$. We have used a similar method that requires off-cardinal point measurements at two zenith angies. Effectively, two independent line-of-sight velocity measurements are obtained from the same emitting region, by assuming that the Earth is rotating under a wind field that is fixed during the time between the measurements. Similar assumptions have been applied for radar mapping of plasma drifts [Evans et al., 1979], and in theoretical models of the thermosphere [Dickinson et al., 1981; Roble et al., 1982]. This technique has been applied to data obtained with the interferometers at Calgary, Alberta and Ann Arbor, Michigan [Meriwether et al., 1983a]. We shall refer to this as the "Calgary approach", and it will be described in detail later in this paper.

We have also developed a method that combines the standard and Calgary approaches. The wind field is assumed to vary linearly with latitude, and to be described by a harmonic Fourier series in longitude. The assumptions of linear (or quasi-linear) spatial gradients and the interchangeability of local time and longitude are both required. In some ways this method simply fits the measurements to an empirical model. In principle, this method allows us to determine all the horizontal gradients of the wind field, and hence the vorticity. The technique has mainly been applied to Arecibo data, with encouraging results, although the latitudinal shear of the zonal wind that is derived often appears to be unrealistically large. We refer to this as the "combined approach", and shall discuss it more fully later.

\section{INSTRUMENTATION}

At Calgary, Ann Arbor, and Arecibo, the basic instrument for thermospheric wind measurements is a Fabry-Perot interferometer. All three of these instruments use $15 \mathrm{~cm}$ diameter etalon plates as the high resolution device. Additionally, the Ann Arbor instrument (MAO I) has medium and low resolution etalons used primarily for dayglow measurements [Cocks and Jacka, 1979; Cocks et al., 1980]. The instruments at Calgary (MAO II) and Arecibo (AAO) use conventional pressure scanning with a single $R C A$ 31034A photomultiplier tube as the detector. MAO I, on the other hand, employs an image plane detector (IPD) similar to the one on the DE-B satellite [Hays et al., 1981; Killeen et al., 1983a; Killeen et al., 1983b]. Using an IPD eliminates the need for pressure scanning of the instrument as light is collected simultaneously across the fringe profile. Another advantage of an IPD is the "self-normalization" of the signal to rapid fluctuations in intensity which may be present because of auroral emissions. Slower variations caused by bright planets or thin clouds crossing the field-of-view are less likely to cause problems. The Calgary interferometer has been upgraded recently to include an IPD. Previous measurements at Calgary were normalized to the signal from a tracking photometer which was bore-sighted with the interferometer. Table 1 compares the details of the three interferometers. Further information about the automatic airglow observatory at Calgary may be found in reports by Meriwether et al. [1983a] and Wickwar et al. [1983].

Detailed data reduction tcchniques that perform the Fourier decomposition of fringe profiles measured by Fabry-Perot interferometers have been described by Hays and Roble [1971b], and in a series of papers by Hernandez [1966, 1970, 1978, 1979]. A simpler reduction technique that we have used for the Arecibo measurements is to fit a Gaussian to the measured fringe profile. This yields results that are sufficiently accurate for neutral wind determinations. For the Calgary and Ann Arbor observations we fit the fringe profile to a Gaussian convolved with the measured instrumental profile [Wick war et al., 1983; Meriwether et al., 1983b].

The zero reference of the instrument is established by using zenith measurements which are made in each cycle of mirror positions. A low order polynomial, such as a cubic, is fit to the zenith data to establish the trend or drift of the instrument. The fitted curve is used as the zero reference for determining the winds in the other observational directions. This method minimises the possible contamination of the zenith reference by shortterm perturbations of the neutral wind field that may be caused by gravity waves propagating through the thermosphere [Hernandez, 1982].

\section{OBSERVATIONS AND ANALYSIS}

Figure 1 illustrates the observing strategy adopted at Calgary and Ann Arbor where off-cardinal point, lineof-sight measurements are made at two zenith angles 
TABLE 1. COMPARISON OF THE PERFORMANCE OF THE THREE DIFFERENT FABRY-PEROT INTERFEROMETERS

\begin{tabular}{|c|c|c|c|}
\hline & MAO I & MAO II & AAO I \\
\hline $\begin{array}{l}\text { Geographic } \\
\text { location }\end{array}$ & $\begin{array}{c}\text { Ann Arbor } \\
42.3^{\circ} \mathrm{N}, 83.7^{\circ} \mathrm{W}\end{array}$ & $\begin{array}{c}\text { Calgary } \\
51.0^{\circ} \mathrm{N}, 114.1^{\circ} \mathrm{W}\end{array}$ & $\begin{array}{c}\text { Arecibo } \\
18.3^{\circ} \mathrm{N}, 67.5^{\circ} \mathrm{W}\end{array}$ \\
\hline Operating mode & Automatic & Automatic & Semi-automatic \\
\hline Detector & ITT $12 \mathrm{ch}$. imaging & GaAs (RCA 31034A) & GaAs (RCA 31034A) \\
\hline Finesse & 11 & 10 & 10 \\
\hline $\begin{array}{l}\text { Typical drift } \\
\text { stability }\end{array}$ & $<5 \mathrm{~m} \mathrm{~s}^{-1}$ per night & $<80 \mathrm{~m} \mathrm{~s}^{-1}$ per night & $<100 \mathrm{~m} \mathrm{~s}^{-1}$ per night \\
\hline $\begin{array}{l}\text { Measurement } \\
\text { time }\end{array}$ & 1 min per fringe & 3 min per fringe & $3.5 \mathrm{~min}$ per fringe \\
\hline $\begin{array}{l}\text { Typical crror } \\
\text { in line-of-sight } \\
\text { vel. (airglow) }\end{array}$ & $30-50 \mathrm{~m} \mathrm{~s}^{-1}$ & $40-60 \mathrm{~m} \mathrm{~s}^{-1}$ & $40-60 \mathrm{~m} \mathrm{~s}^{-1}$ \\
\hline $\begin{array}{l}\text { Typical error } \\
\text { in line-of-sight } \\
\text { vel. (aurora) }\end{array}$ & $10-20 \mathrm{~m} \mathrm{~s}^{-1}$ & $20-30 \mathrm{~m} \mathrm{~s}^{-1}$ & - \\
\hline $\begin{array}{l}\text { Number of points } \\
\text { per map }\end{array}$ & 10 & 10 & 9 \\
\hline $\begin{array}{l}\text { Number of map } \\
\text { cycles per hour }\end{array}$ & 6 & 2 & 1.7 \\
\hline
\end{tabular}

$\left(60^{\circ}\right.$ and $45^{\circ}$ for MAO I, $70^{\circ}$ and $45^{\circ}$ for MAO II). Measurements at cardinal compass headings are not generally made with the exception of the northernmost observations point at Calgary. Standard Arecibo measurements are made at eight azimuth positions (cardinal plus off-cardinal points) and only at $60^{\circ}$ zenith angle, but interweaved with a zenith measurement every $15 \mathrm{~min}$.

After the removal of the instrumental drift by fitting a curve to the peak pressure obtained during the zenith

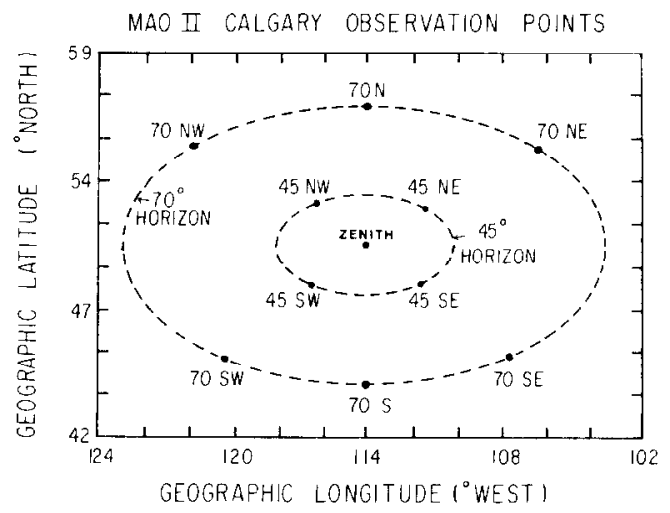

Fig. 1. Schematic ILlustration of the observing program EMPLOYLD AT CALGARY.

The observations at $70^{\circ} \mathrm{N}$ and $70^{\circ} \mathrm{S}$ are used to provide the meridional velocity component only. observations, the pressure shifts as a function of azimuth and zenith angle are converted to horizontal wind velocities. The curvature of the Earth and atmospheric refraction are taken into account in the conversion. However, it is assumed that the vertical wind velocity is zero. It is at this point that the Arecibo analysis differs from that used at the other observing stations. We shall first describe the analysis methods used for Arecibo data.

\section{(1) Arecibo}

The standard approach to calculating mean overhead winds and horizontal velocity gradients at Arecibo has been described by Burnside et al. [1981]. In that paper it was also noted that vorticity could be estimated if local time and longitude were considered to be equivalent. In other words, the "combined approach" described in the Introduction was investigated. Some preliminary results were presented, showing the derived neutral wind field as a function of latitude and local time. We have since developed a procedure to generate maps of the wind field by fitting a model to all the data simultaneously.

The standard approach that we use to analyse Arecibo neutral wind data [Burnside et al., 1981] involves expanding both the meridional and zonal components in a two dimensional Taylor expansion about the zenith. Terms of higher order than those 
representing linear variations are not retained. There are eight measurements in each observation cycle, and the five unknown parameters (which represent the mean flow, its divergence, and its deformation) are determined by the method of least squares. This procedure is then repeated for the next cycle of observations. To employ the "combined approach", we expand the gradient terms in the local Taylor expansion as Fourier expansions in longitude. For example, the zonal velocity component is represented by

$$
\begin{aligned}
u(x, y) & =u_{\mathrm{o}}+a_{\mathrm{o}} x+b_{\mathrm{o}} y \\
& +\sum_{n=1}^{N}\left[a_{n} \sin \left(n x-\alpha_{n}\right) \cdot x+b_{n} \sin \left(n x-\beta_{n}\right) \cdot y\right]
\end{aligned}
$$

where $x$ is longitude and $y$ is the meridional distance from the station to the volume being observed. A similar expansion is made for the meridional component $t(x, y)$. The number of unknown parameters needed to model the wind field by this method is given by $8 N+5$ where $N$ is the number of Fourier components included. For $N=4$, we therefore have 37 unknown parameters, together with about 140 measurements (a typical number for $10 \mathrm{~h}$ of observations). All of the data obtained during a night is therefore analysed by a single run of a least squares fitting procedure. Once the free parameters have been determined, the calculation of the wind velocity field (and the divergence and vorticity fields) is straightforward.

With the nonlinear least squares fitting method, there is some concern over whether a local rather than the global minimum is obtained as the solution. In the actual program an additional stipulation is made that the sum of the residuals falls below a certain value. So long as reasonable starting values for the free parameters are known, the problem of falling into a relative minimum and not the deepest minimum is usually avoided.

\section{(2) Calgary and Ann Arbor}

As we have seen, the Arecibo analysis requires the assumption that wind field variations are linear with latitude. The Calgary approach does not require this constraint on the uniformity of the wind field. The measurements from a particular mirror cycle are not all taken together; only pairs of observations are used to determine the wind vectors. This method preserves the independence of wind observations made at diferent latitudes. The technique is also simpler and requires less computational time.

Maps of the wind field are made using measurements from four "bands" of latitude. Each latitude band is independent of the others, in the sense that no assumptions are made about the structure of the wind from one latitude to the next. The only assumption in this method is that a wind measured at, for example, $70^{\circ} \mathrm{NE}$ is the same as that measured at $70^{\circ} \mathrm{NW}$ a short time later (see Fig. 1). This is the same as assuming that local time and longitude are equivalent. However, the restriction need only be valid for the total time displacement of the two measurements. As the observatory moves along in time under a stationary wind field, an emitting region to the North of the observatory is first viewed when looking to the Northeast at time $t$, and then viewed to the Northwest at a later time $t+\Delta t$. At Calgary, $\Delta t$ is about $1 \mathrm{~h}$ for $70^{\circ}$ zenith angle observations and $20 \mathrm{~min}$ for the measurements at $45^{\circ}$ zenith angle. Clcarly, this procedure may break down during intense substorm activity or during propagation of large scale thermospheric waves [Hernandez and Roble, 1978].

To form the vectors, line-of-sight measurements are first fit with a harmonic series. This procedure provides a means to smooth the data by filtering high frequency components which are treated as noise. The series also enables us to shift the measurements that do not lie along the meridian of the observatory either forward or backward in time. This method therefore references the measured winds to the longitude of the observatory. Next, complementary pairs of line-ofsight observations are combined to calculate the meridional and zonal components. The pairs chosen were $70^{\circ} \mathrm{NE}$ and $70^{\circ} \mathrm{NW}, 70^{\circ} \mathrm{SE}$ and $70^{\circ} \mathrm{SW}$, and similar combinations of the $45^{\circ}$ zenith angle measurements. By using azimuths $45^{\circ}$ from the meridian, the technique has an equal precision in determining the meridional and zonal velocity components. This procedure is further illustrated by Fig. 2, where a Fourier series is fit to each direction of measurement. These series are weighted as a function of the measurement errors.

It is necessary to decide how much smoothing to apply to the time series or data record. The choice of too low an order in the series will smooth the data too much; a high order selection will reflect noise fluctuations in the measurement. To answer this question, we examined the natural fluctuations inherent in the data. The relative power distributed at various harmonic frequencies gives us an indication of the ratio of signal to noise in the measurements. Before fitting the series, a power spectrum is first applied to the set of measurements in each direction. We have used a maximum entropy algorithm to calculate the power spectra. A typical maximum entropy spectrum is shown in Fig. 3. The harmonic peaks in the resulting spectra are examined to determine the amount of signal at higher frequencies relative to the lower frequencies (diurnal and semi-diurnal components). The highest 


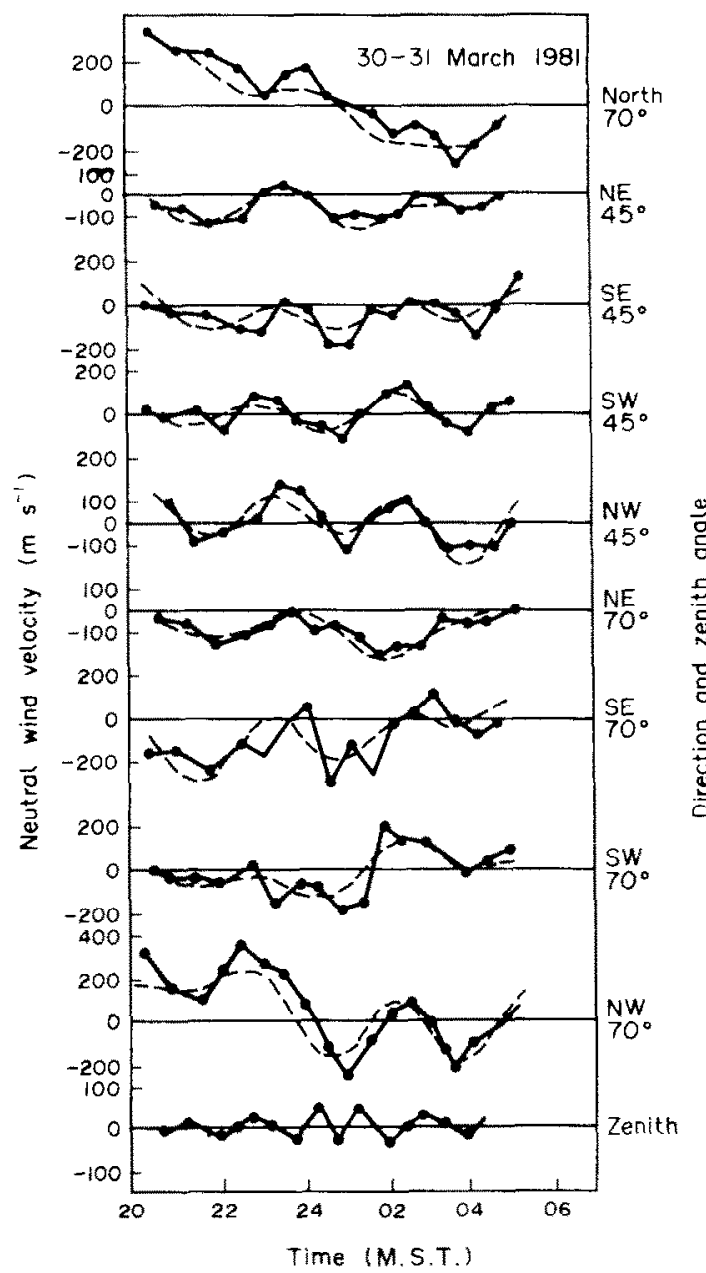

FIG. 2. LINE-OF-SIGHT VELOCITIES OBSER VED IN NINE DIRECTIONS ON 30-31 MARCH 1981.

The measurements to the North at $70^{\circ}$ zenith angle are shown at the top. The next four graphs show the measurements at a zenith angle of $45^{\circ}$, underneath which are the measurements at $70^{\circ}$ zenith angle. The dashed lines are the Fourier fits to these data. The residual vertical velocity obtained after fitting a pulynomial to the zenith measurements is shown at the bottom.

frequency at which significant power appears is used to select the order of the fitted series. This procedure can be easily automated to produce the final wind vector maps.

Figure 4 is a block diagram that summarises the procedure adopted to analyze Fabry- Perot wind data from the Calgary and Ann Arbor instruments.

\section{RESULTS}

(1) Arecibo

While the expansion in (1) would appear to be formally more correct, we find that the fitting procedure
Period (h)

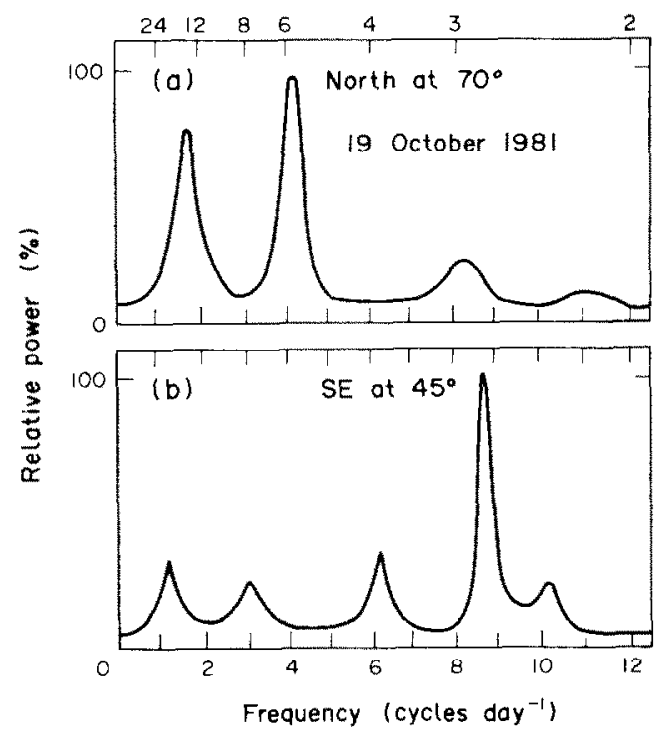

Fra. 3. Two examples of THE power spectra DETER MINFE FROM THE LINE-OF-SIGHT VELOCITY MEASUREMENTS AT Calgary on 19 -20 OCtOBER 1981.

is more stable if multiplication by $x$ is dropped from the fourth term of (1). Longitudinal gradients were therefore modelled simply as $\sin (n x)$ rather than as $x \sin (n x)$. In addition, we find that 37 free parameters are too many to give reliable results. We therefore restricted the Fourier sums in (1) to $N=1$ for the zonal velocity component and $N=3$ for the meridional component. This leaves only 19 free parameters, and we find that this number is generally sufficient to fit the observational data fairly well. A typical result obtained using the combined method to analyse Arecibo data is shown in Fig. 5 a.

We have compared the results obtained from the combined approach with those obtained using the standard method of treating each group of eight measurements separately. Both methods give essentially the same mean wind velocities and the same latitudinal gradients in the meridional wind. However, the latitudinal gradient of the zonal component is not obtained from the standard method, and its determination using the combined method relies heavily on the equivalence of longitude and local time. For this reason we do not place too much credence in the zonal shear that is evident in Fig. 5a.

In order to compare the combined and Calgary approaches, we have used the Calgary method to analyse the measurements made at Arecibo on 16-17 April 1980. The result is shown in Fig. 5b. Only the four measurements at azimuths of $45^{\circ}$ from the cardinal 


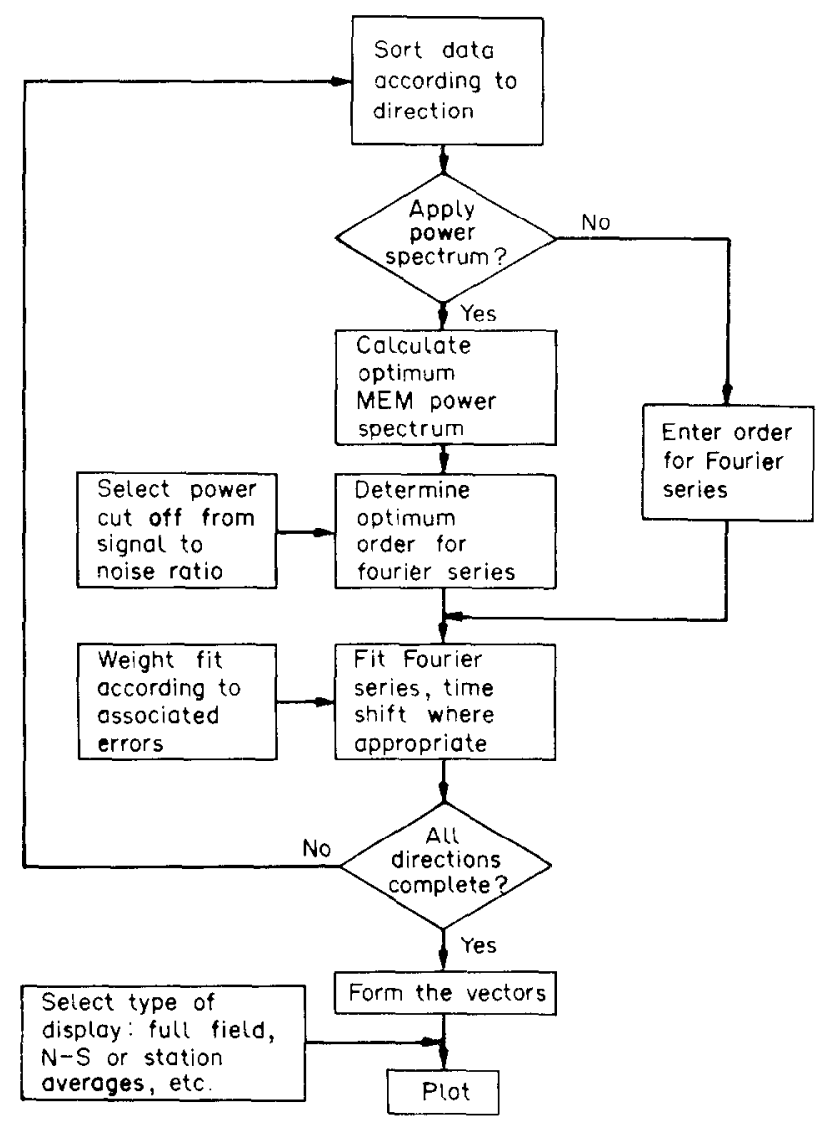

Fig. 4. The procedure used to analyze data from Calgary and Anv Arbor, with the option to use the MAXIMUM ENTROPY METHOD TO DETERMINE THE OPTIMUM ORDER OF THE FOURIER SERIES.

directions were used to produce the map shown in Fig. $5 \mathrm{~b}$, while the measurements at all eight azimuths were used to produce the results in Fig. 5a. For this reason, the two bands of vectors in Fig. 5b cover a smaller latitudinal range than is shown in Fig. 5a. Nevertheless, the flow patterns determined by the two methods are quite similar. The meridional wind convergence near midnight and the divergence near 04.00 AST are reproduced by both methods of analysis. A converging flow in the meridional direction between 00.00 and $02.00 \mathrm{AST}$ is a feature that was observed frequently at Arecibo in the spring and summer of 1980 [Burnside $e t$ al., 1981].

The combined approach has also been applied successfully to analyse the neutral temperature field at Arecibo. However, in this paper we restrict our discussion to the neutral wind measurements.

\section{(2) Calgary and Ann Arbor}

A wind field map produced using the Calgary method of analysis is shown in Fig. 6b, where the vectors derived from the line-of-sight observations shown in Fig. 2, are displayed as a function of time and latitude. The Calgary measurements on this night were also analysed using the combined approach, giving the results that are shown in Fig. $6 \mathrm{a}$.

From Higs. $6 a$ and $6 b$, it is clear that the two methods give similar values for the overhead wind velocities (at $51^{\circ}$ latitude). The results to the North of Calgary are also in reasonable agreement. However, the inferred meridional velocity components to the South of Calgary differ markedly, especially between 20.00 and $00.00 \mathrm{MST}$. The two methods also give rather different zonal velocity components South of Calgary, although the difference is not as pronounced as it is with the meridional component. The reason for this discrepancy may be seen by examining Fig. 6 b. The Calgary method of analysis indicates that, near $21.00 \mathrm{MSl}$, the northward velocity is largest near Calgary and is smaller to both the North and South of the station. Being constrained to a model that allows only a linear variation with latitude for each component of the 


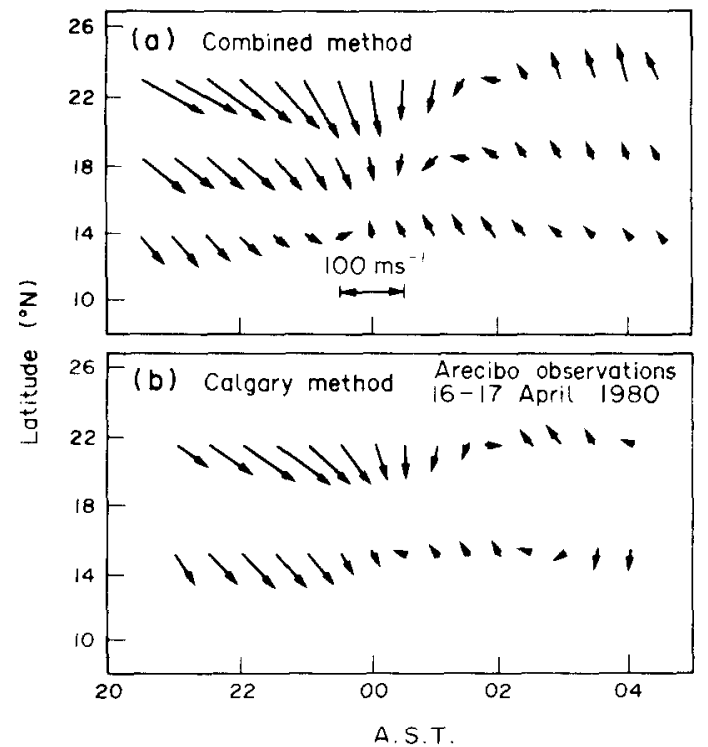

Fig. 5. The WINd FIELd AT Arecibo on 16-17 April 1980, DEDUCED BY USING (a) THE "COMBINED METHOD" AND (b) THE "CAlGary method".

Note that the vectors in (a) cover a slightly greater range of latitude than the vectors in (b).

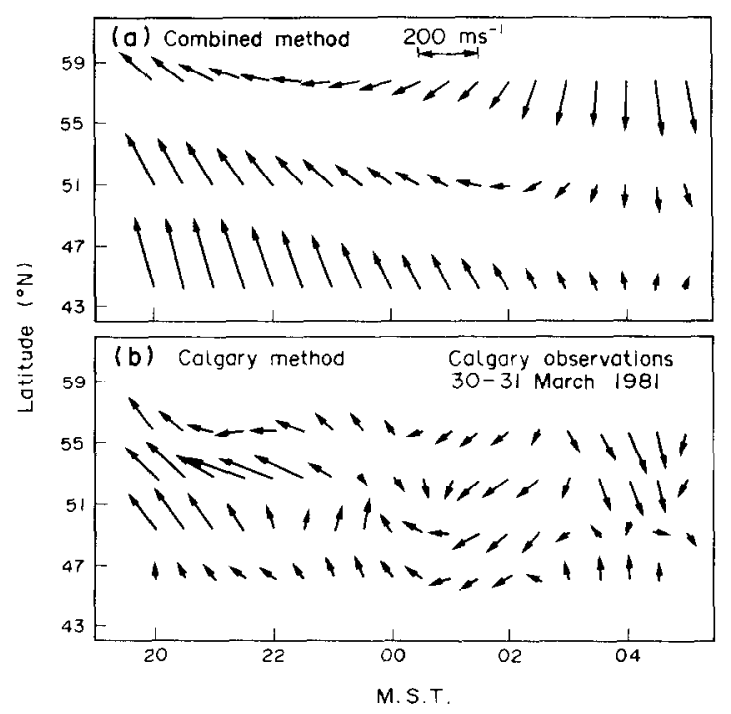

Fig. 6. THE Wind FiEld at Calgary on 30-31 MarCh 1981, DEDUCED BY USING (a) THE "COMBINED METHOD" AND (b) THE "Calgary method".

Although not shown in (b), the measurements to the North at $70^{\circ}$ zenith angle were included in the analysis using the "combined method". The spatial and temporal variations in neutral velocity are considerably greater at Calgary than they are at Arecibo (c.f. Fig. 5). velocity, the combined method cannot reproduce such a wind field. Because of the enhanced airglow intensities at higher latitudes, the most precise velocity measurements are usually those obtained looking northward from Calgary. These measurements are therefore given the most weight in the least squares fit used in the combined method, at the expense of an inaccurate representation of the wind field to the South We also note that the southern measurements are most prone to errors caused by light scattered into the fieldof-view from the brighter northern region. This problem has been studied by Abreu et al. [1983].

Two other types of vector plots have been found useful in the analysis of a night of observations from Calgary. These are the vector averages of measurements to the North and South of the observatory, and a total vector or "station" average of all measurements plotted as a function of time. The first of these plots gives a better indication of the gradients and divergence of the wind field where the statistics are inadequate to display this information in the full vector field representation. The second plot is yet another way to improve statistics by averaging all measurements at a particular time into a single vector. The station averages are useful for displaying many nights of data and for comparing data from several observatories. A comparative examination of the Calgary and Ann Arbor data base is presented by Meriwether et al. [1983b].

\section{DISCUSSION}

It appears that both the experimental methods that we have described have their place in the study of thermospheric neutral winds. For the analysis of measurements made at Arecibo, both the "combined approach" and the "Calgary approach" give similar results (Fig. 5). Although the Arecibo observing scheme is not optimum for use with the Calgary method of analysis (and only half the measurements were utilised in the analysis by the Calgary method), the agreement is fairly good. It therefore seems that the description of the wind field implicit in the Arecibo combined approach is a reasonable approximation for low-latitudes.

However, the combined approach often does not model the wind field at Calgary very well (Fig. 6). We attribute this to spatial and temporal variations in neutral velocity (and emission intensity) that are considerably greater at Calgary than they are at Arecibo. Therefore, the lack of assumptions about spatial gradients in the wind field is an attractive feature of the "Calgary approach". In principle, the "combined approach" could be extended by adding another term, 
representing a quadratic variation with latitude, to the Taylor expansion for the velocity components. Such an approach might be especially fruitful if measurements were a vailable from two or more independent stations, separated by some hundreds of kilometres. However, for the routine analysis of the Calgary observations there seems to be nothing to gain from using the "combined approach".

It is hard to determine the accuracy of the assumption that longitude and local time are equivalent. However, a study made by Hernandez $\mathrm{et} \mathrm{al}$. [1978] using two mid-latitude stations separated by $26^{\circ}$ in longitude does provide some support for this assumption. The applicability of this assumption presumably varies, even during the course of a night. By calculating station averages, some of the errors introduced by breakdowns in this assumption might be expected to cancel. Such results are given by Meriwether et al. [1983a,b].

The power spectrum used in the Calgary data analysis may be used to investigate the degree of periodic oscillations inherent in the wind measurements. Here we have simply used the power spectrum as a numerical tool to automate the selection of the order of fitted harmonic series. However, a logical extension to the problem might be the examination of the data in terms of the relationship between harmonic frequencies of the winds and real geophysical parameters.

As instrumentation continues to improve, future progress in the study of thermospheric dynamics will be possible because of improved sampling of the thermospheric wind field, using methods such as we have described in this paper. The assumptions that are necessary in the analysis of observations obtained by a single station would not be necessary with a bistatic observatory in which two instruments were employed to observe a common volume. Such an observatory would allow us to make a critical test of the assumption that local time and longitude are equivalent (Rees and Greenaway, 1983). Two stations at the same latitude, and separated by some $750 \mathrm{~km}$, could scan the common meridian between the stations and thus measure the latitudinal shear of the zonal wind and the vorticity of the wind field directly.

Acknowledgments - We thank Allan Clark of the Rothney Astrophysical Observatory, University of Calgary, for providing the site at Calgary. This work was supported by grants ATM80-15316, ATM81-16749 and ATM82-02440 from the Atmospheric Research Section, National Science Foundation, and by NASA grant NASW-32 to the University of Michigan. The Arecibo Observatory is operated by Cornell University under contract with the National Science Foundation.

\section{REFERENCES}

Abreu, V. J., Schmitt, G. A., Hays, P. B., Meriwether, J. W., Tepley, C. A. and Cogger, L. L. (1983). Atmospheric scattering effects on ground-based measurements of the thermospheric winds. Planet. Space Sci. 31, 303.

Browning, K. A. and Wexler, R. (1968). The determination of kinematic properties of a wind field using Doppler radar. J. appl. Meteor. 7, 105.

Burnside, R. G., Herrero, F. A., Meriwether, J. W. and Walker, J. C. G. (1981). Optical observations of thermospheric dynamics at Arecibo. J. geophys. Res. 86, 5532.

Cocks, T. D. and Jacka, F. (1979) Daytime thermospheric temperatures, wind velocities and emission intensities derived from ground based observations of the OI $6300 \mathrm{~nm}$ airglow line profile. J. atmos. terr. Phys. 41, 409.

Cocks, T. D., Creighton, D. F. and Jacka, F. (1980) Application of a dual Fabry-Perot spectrometer for daytime airglow studies. J. atmos. terr. Phys. 42, 499.

Dickinson, R. E., Ridley, E. C. and Roble, R. G. (1981). A threedimensional general circulation model of the thermosphere. J. geophys. Res. 86, 1499.

Evans, J. V., Holt, J. M. and Wand, R. H. (1979) Millstone Hill incoherent scatter observations of auroral convection over $60^{\circ} \leqslant \Lambda \leqslant 75^{\circ}$. 1. Observing and data reduction procedures. J. geophys. Res. 84, 7059.

Hagfors, T. and Behnke, R. A. (1974). Measurement of threedimensional plasma velocities at the Arecibo Observatory. Radio Sci. 9, 89.

Hays, P. B. and Roble, R. G. (1971a). Direct observations of thermospheric winds during geomagnetic storms. J. geophys. Res. 76, 5316.

Hays, P. B. and Roble, R.G.(1971b) A technique for recovering Doppler linc profiles from Fabry-Pcrot interferometcr fringes of very low intensity. Appl. Opt. 10, 193.

Hays, P. B., Killeen, T. L. and Kennedy, B. C. (1981). The Fabry-Perot interferometer on Dynamics Explorer. Space Sci. Inst. 5, 395.

Hernandez, G. (1966). Analytic description of a Fabry-Perot photoelectric spectrometer. Appl. Opt. 5, 1745.

Hernandez, G. (1970). Analytic description of a Fabry-Perot photoelectric spectrometer. 2 : Numerical results. Appl. Opt. 9. 1591.

IIernandez, G. and Roble, R. G. (1976). Direct measurements of thermospheric winds and temperatures, 1, Seasonal variations during geomagnetically quiet periods. $J$. geophys. Res. 81, 2065.

Hernandez, G. (1978). Analytical description of a Fabry-Perot spectrometer. 4: Signal noise limitations in data retrieval; winds, temperature and emission rate. Appl. Opt. 17, 2967.

Hernandez, G. (1979). Analytical description of a Fabry-Perot spectrometer. 5: Optimization for minimum uncertainties in the determination of Doppler shifts and widths. Appl. Opt. 18,3826 .

Hernandez, G. and Roble, R. G. (1978). Observations of largescale thermospheric waves during geomagnetic storms. $J$. geophys. Res. 83, 5531.

Hernandez, G., Sipler, D. P. and Biondi, M. A. (1978). Simultaneous measurements of midlatitude thermospheric winds at $105^{\circ} \mathrm{W}$ and $79^{\circ} \mathrm{W}$ longitude. Geophys. Res. Lett. 5 , 935.

Hernandez, G. and Roble, R. G. (1979). Thermospheric dynamics investigations with very high resolution spectrometers. Appl. Opt. 18, 3376.

Hernandez, G. (1980). Measurement of thermospheric 
temperatures and winds by remote Fabry-Perot spectrometry. Opt. Eng. 19, 518 .

Hernandez, G. (1982). Vertical motions of the neutral thermosphere at mid-latitudes. Geophys. Res. Lett. 9, 555.

Killeen, T. L., Hays, P. B., Spencer, N. W. and Wharton, L. E. (1982). Neutral winds in the polar thermosphere as measured from Dynamics Explorcr. Geophys. Res. Lett. 9 , 957.

Killeen, T. L. and Hays, P. B. (1983a). Doppler line profile analysis for a multi-channel Fabry-Perot interferometer. Appl. Opt. (in press).

Killeen, T. L., Hays, P. B., Kennedy, B. C., Symanow, D. A. and Ceckowski, D. H. (1983b). An image plane detector for the Dynamics Explorer Fabry-Perot interferometer. Appl. Opt. (in press).

Meriwether, J. W. (1983). Observations of thermospheric dynamics at high latitudes from ground and space. $R$ ad. $S c i$. (in press).

Meriwether, J. W., Tepley, C. A., Price, S. A., Hays, P. B. and Cogger, I. I.. (1983a). Remote ground-based observations of terrestrial airglow emissions and thermospheric dynamics at Calgary, Alberta, Canada. Opt. Eng. 22, 128.
Meriwether, J. W., Tepley, C. A., Hays, P. B. and Cogger, L. L. (1983b). Thermospheric neutral wind measurements over Calgary and Ann Arbor. Planet. Space Sci. (to be submitted).

Rees, D. and Greenaway, A. H. (1983). Doppler imaging systems; an optical device for measuring vector winds. 1: General principles. Appl. Opt. 22, 1078.

Roble, R. G., Dickinson, R. E. and Ridley, E. C. (1982). Global circulation and temperature structure of thermosphere with high-latitude plasma convection. J. geophys. Res. 87, 1599.

Sipler, D. P. and Biondi, M. A. (1979). Midlatitude F region neutral winds and temperatures during the geomagnetic storm of March 26, 1976. J. geophys. Res. 84, 37.

Smith, R. W., Sivjee, G. G., Stewart, R. D., McCormac, F. G. and Deehr, C. S. (1982). Polar cusp ion drift studies through high resolution interferometry of $\mathrm{O}^{+} 7320 \AA$ emission. $J$. geophys. Res. 87, 4455.

Wickwar, V. B., Meriwether, J. W., Hays, P. B. and Nagy, A. F. (1983). Comparison of simultaneous and averaged measurements of the meridional thermospheric neutral wind by radar and optical techniques in the auroral region. $J$. geophys. Res. (in press). 